C1998 IEEE. Personal use of this material is permitted. However, permission to reprint/republish this material for advertising or promotional purposes or for creating new collective works for resale or redistribution to servers or lists, or to reuse any copyrighted component of this work in other works must obtained from the IEEE.

\title{
Low Cost Transmission Line Demonstrator For A Novel Intermediate Level Communication Engineering Laboratory
}

F. Torres, Member, IEEE, I. Corbella, A. Cardama, Member, IEEE, J. Romeu, Member, IEEE, O. Galíndez

Abstract - This paper is devoted to the description of a transmission line laboratory demonstrator for intermediate level communication engineering students, within a course on Radiation and Guided Waves. Student activities have been organized according to the needs of intermediate level students and the objectives of an introductory laboratory, which are substantially different from those of specialized laboratory activities aimed at higher level students and placed just before graduation. Since a large number of students attend these laboratory sessions before they choose their specialty, low cost is an unavoidable requirement to be considered. For this reason, the measurement set-up makes use of standard instrumentation, usually available in a basic electronic laboratory, which allows multiplication of work places at a reasonable cost.

Both time domain reflectometry and sinusoidal steady state measurements are presented to characterize the main parameters of a coaxial line. However, discussions are mainly focused on sinusoidal measurements, which are scarce in the literature and exclusively devoted to slotted line measurements. The experimental work undertaken by the students gives them insight into main transmission line parameter and features: line impedance, propagation speed, its behavior as an impedance transformer, the complex nature of the measured magnitudes, the standing wave behavior of voltage, and attenuation.

\section{Introduction}

The Universitat Politècnica de Catalunya (UPC) was founded in 1971 by the merging of existing engineering and architecture schools in Barcelona, Spain. At present, the UPC has over 30,000 students enrolled in technical studies such as civil engineering, 
architecture, computer science, and mechanical and electrical engineering. The Telecommunication Engineering School was created in 1971, and since then it has been steadily growing up to its present size of approximately 2,800 undergraduate students and 230 instructors. Since 1992, the curriculum of Telecommunication Engineering has been in process of reform, within the frame of an overall plan whose main objectives are: 1) inclusion of the new technologies, 2) increase in the flexibility of students' individual curriculum, and 3) increase in the percentage of experimental work.

In this context, the laboratory sessions object of this paper are included as part of a course on Radiation and Guided Waves, taken by the students at their fifth semester. The first part of this subject (70\%) is devoted to the principles of transmission lines, waveguides and fiber optics. The second part (30\%) presents antenna parameters and propagation principles. Each semester about 240 students take this course, prior to their specialization. Hence, multiplication of workbenches was mandatory and cost was an unavoidable parameter taken into account.

\section{Objectives and Laboratory Description}

The laboratory consists of an external part with the antennas (Fig. 1) and a room with 10 work benches that can accommodate two students each. Thus, 20 students can work simultaneously, every two-hour session. In order to be synchronized to the lectures in Radiation and Guided Waves, these two-hour sessions are arranged along the semester as follows:

1) TV-TVSAT distribution network

2) Pulse propagation in transmission lines

3) Sinusoidal steady state in transmission lines

4) Slotted rectangular wave-guide measurements at $X$ band

5) Antenna polarization measurements

6) Antenna radiation pattern characterization 


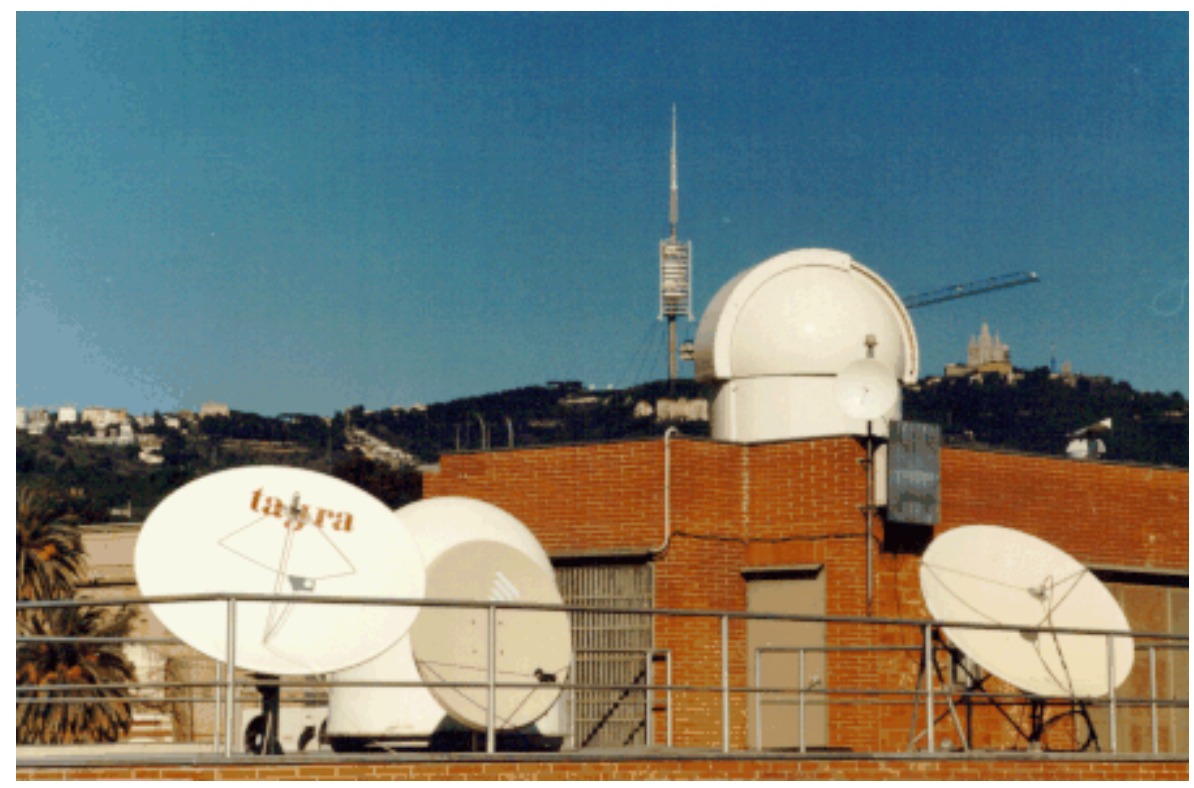

Fig. 1. Roof of the laboratory building showing several antennas used for some of the experiments.

During the weeks previous to each two-hour session the students attend lectures and do a wide variety of exercises related to the topic. Before each session, the students are assigned homework consisting of some theoretical or numerical exercises directly related to the experimental work. By doing so, the students do not have to perform time consuming theoretical analyses or data manipulation in the laboratory. Moreover, all expressions which are required to analyze experimental data and theoretical results have been developed in advance in order to make the comparison between theoretical and measured data straightforward. This increases the time the students spend in the laboratory performing measurements, thinking about the measurement principles, and interpreting results.

As shown in the next sections, measurement set-up principles, task descriptions, and blanks to be filled with experimental data are mixed in the text. This forces the students to read the text carefully (they are expected to have done so before the laboratory session) in order to find out what to do and what is expected out of each single measurement. The students finish each session by giving the teacher a form that includes a summary of main results.

Here, it is important to point out that, contrary to current use in laboratory activities, no further report is required. The reasons for this must be clearly explained: By not being asked to write and hand-in a final report on their laboratory activities, students are forced to prepare their homework assignment carefully in advance, in order to take the maximum profit of their experimental activities. Note that the students know that they are going to be evaluated out of their work during the laboratory session, and that there is no further margin to correct, make-up or re-analyze results. Moreover, by doing so, all laboratory homework carried out by the students is devoted to the following laboratory session, and not to the previous one. Of course, we agree that the elaboration of a report after a laboratory activity is a mandatory task in real-life engineering, and that students must be well trained in this sense. However, we think that this valuable training is beyond the scope of an introductory laboratory. In our opinion, how to 
elaborate a technical report should be taught at a specialized laboratory, usually placed at the end of the last semesters, when the students have already acquired more global and mature engineering skills.

As discussed before, the laboratory sessions presented in this paper are devoted to introducing the students into experimental activities. In this sense, each laboratory session is divided into three consecutive sets of measurements with different purposes:

a) Qualitative measurements, without writing down any result, to get insight into the measurement set-up and session objectives.

b) Approximate measurements. Data is compared to theoretical predictions that take into account an ideal behavior of the transmission line.

c) Accurate measurement. Data is compared to theoretical results that take into account parameters that the students have measured along their previous laboratory activities.

\section{Measurement Set-Up}

In order to illustrate the laboratory sessions, this work describes topics 2) and 3), which make use of the same measurement set-up (Fig. 1). The time domain set-up is used to illustrate speed of propagation and attenuation measurements. At the steady state part, the students perform measurements at the frequencies that make the electrical length of a coaxial line to be $\lambda / 2$ or $\lambda / 4$. This allows characterizing basic parameters of the transmission line and highlights its effect as impedance transformer. The set-up permits relative phase and amplitude measurements of a sinusoidal voltage across a reactive load by means of a standard oscilloscope. In this way the students get insight into the phasor nature of the involved magnitudes. This very low cost workbench allows measurements with enough accuracy to measure the attenuation of the line. 


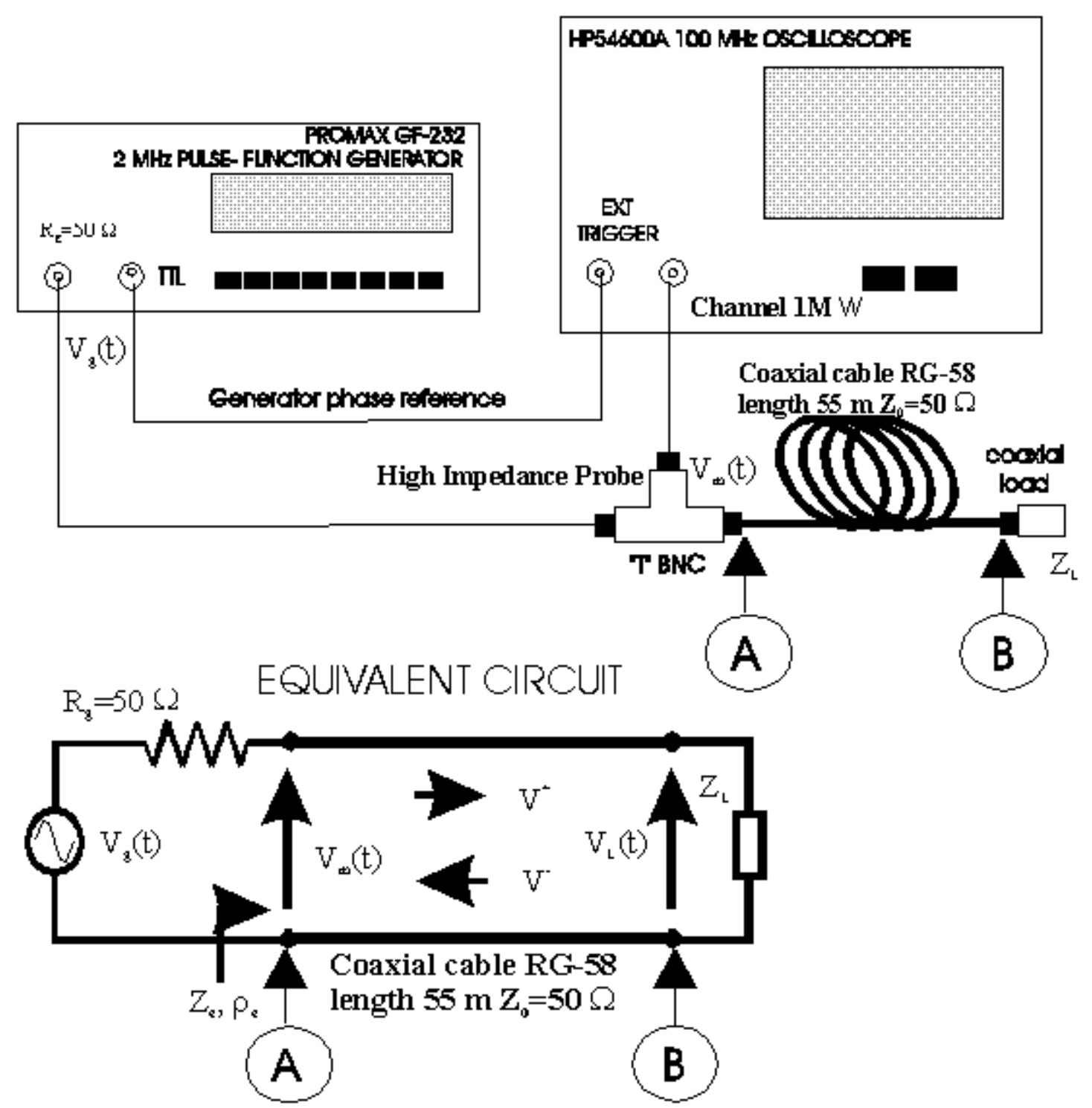

$\mathrm{V}_{\mathrm{c}}(\mathrm{t})$ : gen erat or open circuit voltage $\mathrm{V}_{\mathrm{x}}(\mathrm{t})$ : measured signal at coaxial input

$Z_{\mathrm{e}}$ : Input inpedance of the loaded coaxial line $Z_{L}$ : coaxial load (open, short, matched)

Fig. 2. Laboratory set-up (consisting of a sine-wave generator, an oscilloscope and a coaxial cable) and its equivalent circuit (a transmission line of length 1)

Fig. 2 shows the scheme of the laboratory set-up and its equivalent circuit. It consists of a $50 \Omega$ function generator capable of generating sinusoidal signals up to $2 \mathrm{MHz}$. It is connected to a 55 meters coaxial cable through a BNC "T" connector. This allows to connect a high impedance probe $(1 \mathrm{M} \Omega)$ to measure the voltage at the line input without perturbing the transmitted and received signal. The length of the cable has been chosen to have an electrical length slightly over $\lambda / 2$ when using the highest generator frequency available. Some BNC terminations are also required to load the coaxial line: a short circuit, a matched load, an open circuit, a $75 \Omega$ load, and some reactive loads. The coaxial cable used in this experiment has the typical parameters shown in Fig. 3, as given by the manufacturer. Attenuation $(\mathrm{dB} / 100 \mathrm{~m})$ has been accurately measured by means of a network analyzer in order to allow a good comparison of measured data to experimental results. Note that external triggering is required to have a reference of the generator phase to perform phase relative measurements on the sinusoidal steady state 
section. No amplitude calibrations are required because all measurements are relative.

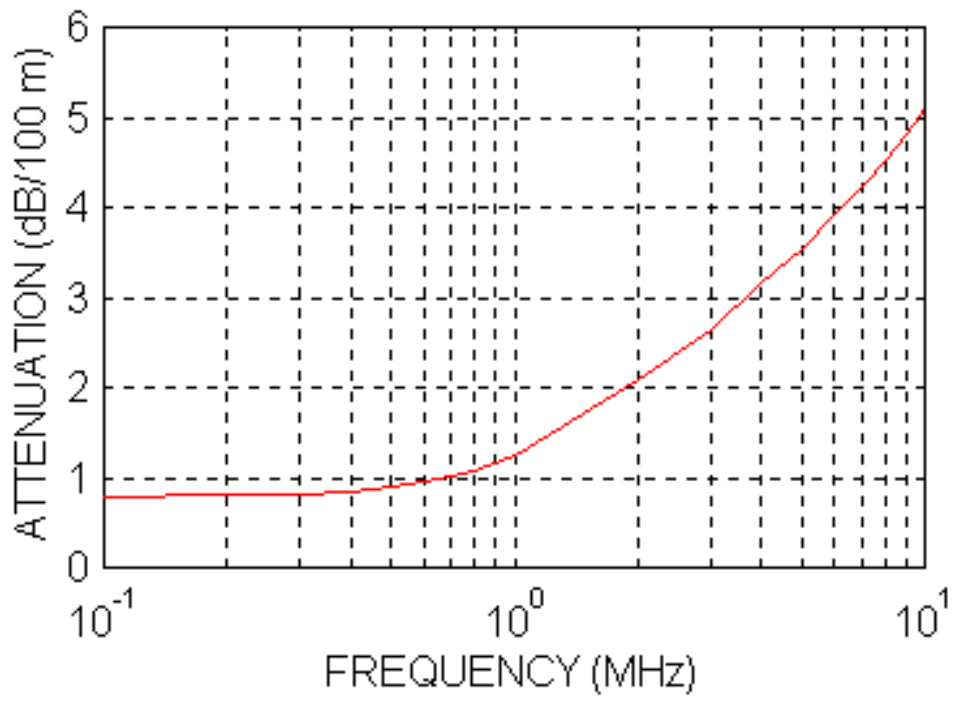

RF coaxial cable RG $58 \mathrm{c} / \mathrm{u}$ single screened.

Impedance $50+/-2 \Omega$ Capacitance $101 \mathrm{pF} / \mathrm{m}$. Velocity of signal $66.2 \%$ of speed of light.

Fig. 3: Coaxial cable attenuation graph $(\mathrm{dB} / 100 \mathrm{~m})$ obtained by means of a network analyzer.

The following sections describe the measurements as given to the students in their laboratory notebooks. Underlined data are typical measurements performed by the students. In the notebooks these underlined data are substituted by blanks to be filled in by the students. Theoretical expressions are derived by the students by means of exercises proposed by the teacher in sessions previous to the laboratory work. In this way laboratory time is optimized in the sense that students spend their whole time performing the measurements and analyzing the experimental data. Along this paper some oscilloscope displays are shown in order to illustrate the measurements and justify the calculations They have been obtained by scanning the hard copy oscilloscope output, and the authors apologize for their lack of quality, which is the result of reducing the file sizes, in order to speed up their view on the web. The oscilloscopes had not the capability of creating .jpg or other image format files.

\section{Time domain measurements}

As this issue is well known, we just summarize some basic results that are used by the students in the steady state section. Fig. 4 shows the oscilloscope screen when the coaxial line output is in open circuit while a $400 \mathrm{~ns}$ pulse is fed at the line input at a 500 $\mathrm{kHz}$ rate. 


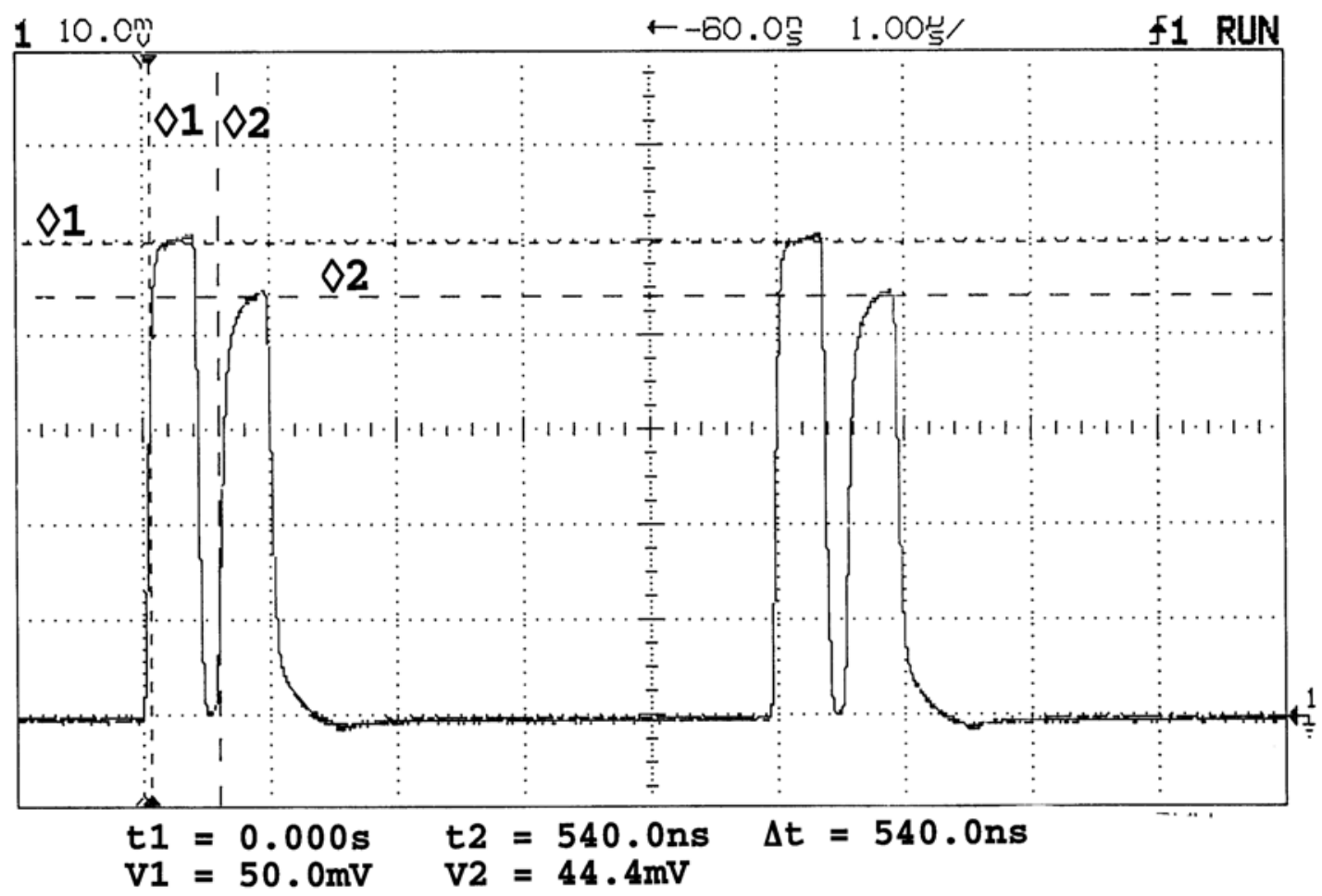

Fig. 4: Plot of a $400 \mathrm{~ns}$ train of pulses injected at a $500 \mathrm{kHz}$ rate, and the reflection of every single pulse.

The students realize that the generator is well matched to the line because there is only one reflection of every transmitted pulse. They measure de amplitude of generator open circuit voltage $\mathrm{V}_{\mathrm{oc}}$, of the transmitted pulse $\mathrm{V}_{\mathrm{e}}^{+}$, of the reflected pulse $\mathrm{V}_{\mathrm{e}}^{-}$, and the reflection delay:

$$
\mathrm{V}_{\mathrm{oc}}=105.1 \mathrm{mV} \mathrm{V} \mathrm{e}^{+}=50 \mathrm{mV} \mathrm{V}{ }_{\mathrm{e}}^{-}=44.4 \mathrm{mV} \Delta \mathrm{t}=540 \mathrm{~ns}
$$

Taking into account that the generator internal impedance is $\mathrm{R}_{\mathrm{g}}=50 \Omega$, the line impedance is calculated as

$$
Z_{o}=\frac{V_{e}^{+}}{V_{e}^{+}+V_{o c}} R_{\mathrm{g}}=55.1 \mathrm{Ohm}
$$

Note that this value is larger than the $Z_{0}=50 \pm 2 \Omega$ specification. This is because the cable is of bad quality and does not comply with the specifications. It must be pointed out that the students are very surprised by this result. In fact a large percentage of them repeat the measurement until they get a value of about $Z_{0}=51.9 \Omega$ compliant with specs. The relative propagation speed $\mathrm{v}_{\mathrm{p}}$ is obtained out of the propagation delay $\Delta \mathrm{t}$ as:

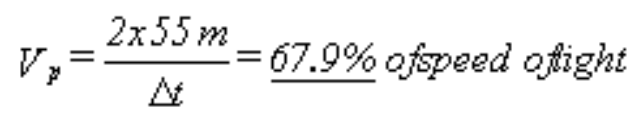

Attenuation in $\mathrm{dB} / 100 \mathrm{~m}$ is calculated as 


$$
A(d B / 100 \mathrm{~m})=20 \log \left(\frac{V_{e}^{+}}{V_{e}}\right) \frac{100}{2 l}=\underline{0.94 \mathrm{~dB} / 100 \mathrm{~m}}
$$

$l$ being the length of the cable. Note that pulse attenuation belongs almost completely to that of the transmitted pulse sequence first harmonic. Since the pulse sequence has a frequency of $0.5 \mathrm{MHz}$, this value matches very well to the plot obtained by means of a network analyzer (Fig. 3).

\section{Sinusoidal Steady-State}

The students start their work by performing some qualitative measurements in order to get used to the laboratory set-up, and the measuring principles. Afterwards, students perform quantitative measurements and compare measured data to that given by cable manufacturer.

\section{A. Voltage Measurements in Sinusoidal Steady State}

The generator open circuit voltage $\left(\mathrm{V}_{\mathrm{g}}(\mathrm{t})\right.$ in Fig. 2) can be measured by disconnecting the $55 \mathrm{~m}$ coaxial cable from the BNC "T" (letting point A in open circuit). Connecting the $50 \Omega$ load to the generator's output -point A- the measured voltage decreases about half of the previous value. In this way it is checked that the generator internal impedance is $R_{g}=50 \Omega$. Now the coaxial cable is inserted between the $50 \Omega$ load and the generator in order to check that the measured voltage has not changed significantly because the cable impedance is nominally $50 \Omega$, and loaded with a matched impedance. Then a short-circuit is connected to the generator output and the measured voltage is, of course, zero. However, if the coaxial line is inserted between the short circuit and point A there appears a signal. By manually sweeping the generator frequency the students notice that the level of the voltage at the input of the line changes. It is also shown that at very low frequencies the effect of the transmission line is negligible, the voltage at point $\mathrm{A}$ is zero because the line is much shorter than the wavelength.

Now, the students sweep the frequency until the measure a voltage maximum. At this point the line is unplugged from the generator output to notice that the measured voltage hardly changes because the line has transformed the short circuit into an open circuit. At this frequency the line has become an impedance inverter, so its electric length is $\lambda / 4$. This allows calculating the permittivity $\varepsilon_{\mathrm{r}}$ of the dielectric:

$$
\begin{gathered}
f=\frac{853 \mathrm{kHz}}{4} \quad \ell=\frac{\lambda}{4}=\frac{c_{0}}{4 f \sqrt{6}} \quad \therefore=\left[\frac{c_{0}}{4 f l}\right]^{2}=\underline{2.55} \\
v_{p}=62.6 \% \text { of speed of light }
\end{gathered}
$$

where $\mathrm{c}_{0}$ is the vacuum velocity of light. Now, if the frequency is increased until a minimum voltage is measured, the line length will be $\lambda / 2$, since the load will be electrically moved to the line input. Another measurement of the electric permitivity can be done: 


$$
\begin{gathered}
f=\underline{1775 \mathrm{kHz}} \quad \ell=\frac{1}{2} \quad \therefore=\underline{2.36} \\
v_{p}=65.1 \% \text { of speed of light }
\end{gathered}
$$

Note that both measurements should give the same value. However, the results show a small difference, and they also are slightly below the value measured in the time domain section.

\section{B. Standing Wave Ratio Measurement}

When a resistive load $\mathrm{R}_{\mathrm{L}}$ different from $\mathrm{Z}_{0}$ is connected at the end of the line, a standing wave appears in it. Thus, if we would move a probe along the line (by means of a slotted line), the measured voltage will have maxima and minima. For an ideal line this is equivalent to let the probe fixed at the line input and change the frequency. In other words, this is another way to change the electrical distance between the probe and the load expressed in wavelengths.

At the workbenches, an unknown load $\mathrm{R}_{\mathrm{L}}(75 \Omega)$ is connected to the coaxial line end. The generator frequency is swept to measure the maximum and the minimum voltage values. The $\mathrm{R}_{\mathrm{L}}$ value can now be determined from the voltage standing wave ratio. However, measuring this ratio is not enough to decide whether the load is larger or lower than $\mathrm{Z}_{0}$. To eliminate this ambiguity, the students select a frequency to have a voltage minimum and then exchange the unknown load with a short-circuit. If a minimum voltage is still measured, the distance to the load is $\lambda / 2$ and $\mathrm{R}_{\mathrm{L}}<\mathrm{Z}_{0}$. On the contrary, if a maximum of voltage is measured the line length is $\lambda / 4$ and $\mathrm{R}_{\mathrm{L}} \mathrm{Z}_{0}$. Fig. 5 shows the voltages measured at the maximum and minimum positions, which gives:

$$
\begin{gathered}
V_{\text {max }}=\underline{120 \mathrm{mV}} ; V_{\text {min }}=\underline{91.25 \mathrm{mV}} \\
S=V_{\text {max }} / V_{\text {min }}=\underline{1.31} R_{L}=S Z_{0}=\underline{72 \Omega}
\end{gathered}
$$




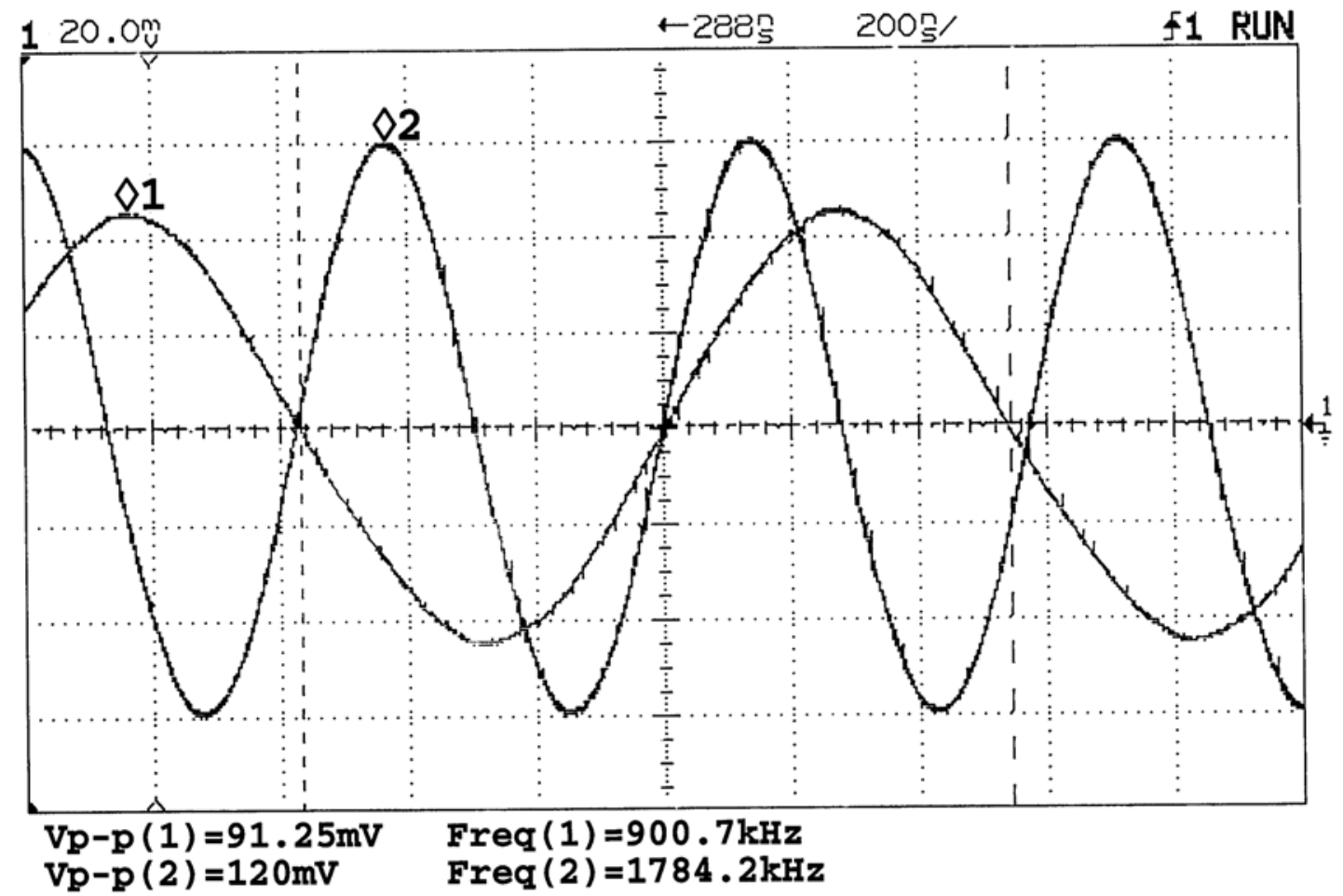

Fig. 5: Standing wave measurement: minimum and maximum amplitude waves obtained by sweeping the signal frequency. The line is terminated with and unknown load $\mathrm{R}_{\mathrm{L}}$ to be determined.

The value used for $Z_{0}$ is that measured in IV $\left(Z_{0}=\underline{55.1} \Omega\right)$. However, note that the measured value is slightly below the expected one. The students are made conscious that this effect is mainly due to line losses, because the SWR is actually measured at the line input, not at the $\mathrm{R}_{\mathrm{L}}$ plane. Losses make the reflection coefficient to reduce as it gets away from the load $\mathrm{R}_{\mathrm{L}}$. Thus, the measured impedance at the line input tends to $\mathrm{Z}_{0}$. This effect is used in section $\mathrm{D}$ to measure the line attenuation.

\section{Complex measurements at the line input}

In this section, the complex voltage at the input of the transmission line ( $\mathrm{V}_{\mathrm{m}}$ in Fig. 2$)$ is measured for three different loads $Z_{\mathrm{L}}$ : a matched load, a short circuit, and an open circuit. The voltage at the line input depends on the load $Z_{\mathrm{e}}$, which is the impedance seen from the generator. The students set the generator to the frequency which makes the electrical length of the line be $3 \lambda / 16$. Now, the value of the input impedance $Z_{e}$ can be calculated taking into account each value of the load $\mathrm{Z}_{\mathrm{L}}$ :

$$
\begin{gathered}
Z_{L}=Z_{0} ; \quad Z_{e}=Z_{0} \\
Z_{L}=0 ; \quad Z_{e}=j Z_{0} \operatorname{tg}(\beta l)=j 2,4 l Z_{0} \\
Z_{Z}=\infty ; Z_{e}=-j Z_{0} \cot g(\beta l)=-j 0.41 Z_{0}
\end{gathered}
$$


where $\beta$ is the phase constant $\beta=\omega / \mathrm{c}$ being $\mathrm{c}$ the velocity of propagation in the line and $\mathrm{Z}_{0}$ the characteristic impedance of the line. The complex voltage at the line input is given by

$$
V_{m}=V_{g} \frac{Z_{e}}{Z_{e}+R_{g}}=\left|V_{m}\right| e^{\eta_{m}}
$$

We consider that the time domain expressions of both voltages are $V_{g}(t)=V_{g} \sin (\omega t)$ and $V_{m}(t)=\left|V_{m}\right| \sin \left(\omega t+\varphi_{m}\right)$. The sinus factor has been chosen in order to have a voltage null associated to the phase $\varphi_{\mathrm{m}}$, which is easier to visualize in the screen. Before going ahead, the frequency at which the line has a length of $3 \lambda / 16$ must be calculated. Taking and average value for the permitivity:

$$
f \frac{3 \mathrm{co}_{0}}{162 \sqrt{c_{\gamma}}}=\underline{0.652 \mathrm{MHz}}
$$

and the generator open circuit voltage measured at this frequency in a sample measurement is (different values will be obtained dependig on the amplitude control knob of the generator):

$$
\mathrm{V}_{\mathrm{g}}=\underline{93 \mathrm{mV}}
$$

Now, the coaxial line is connected to point A, and it is terminated with different loads. The predicted value of $\left|\mathrm{V}_{\mathrm{m}}\right|$ is computed for each load taking into account the measured open circuit voltage $\mathrm{V}_{\mathrm{g}}$ (in this example $93 \mathrm{mV}$ ); and $\left|\mathrm{V}_{\mathrm{m}}\right|$ is measured by connecting the line and terminating it with the corresponding $\mathrm{Z}_{\mathrm{L}}$. Fig. 6 shows the three plots that

\begin{tabular}{|c|c|c|}
\hline load & predicted $\left|\mathbf{V}_{\mathbf{m}}\right|$ & measured $\left|\mathbf{V}_{\mathbf{m}}\right|$ \\
\hline plot $1 Z_{L}=Z_{0}$ & $46.5 \mathrm{mV}$ & $48.7 \mathrm{mV}$ \\
\hline plot $2 Z_{L}=0$ & $85.5 \mathrm{mV}$ & $79.7 \mathrm{mV}$ \\
\hline $\operatorname{plot} 3 Z_{L}=\infty$ & $\underline{35.3 \mathrm{mV}}$ & $31.2 \mathrm{mV}$ \\
\hline
\end{tabular}
have been obtained. $\left|\mathrm{V}_{\mathrm{m}}\right|$ corresponds to the peak value of each sinusoid (this data is not shown in Fig. 6). 


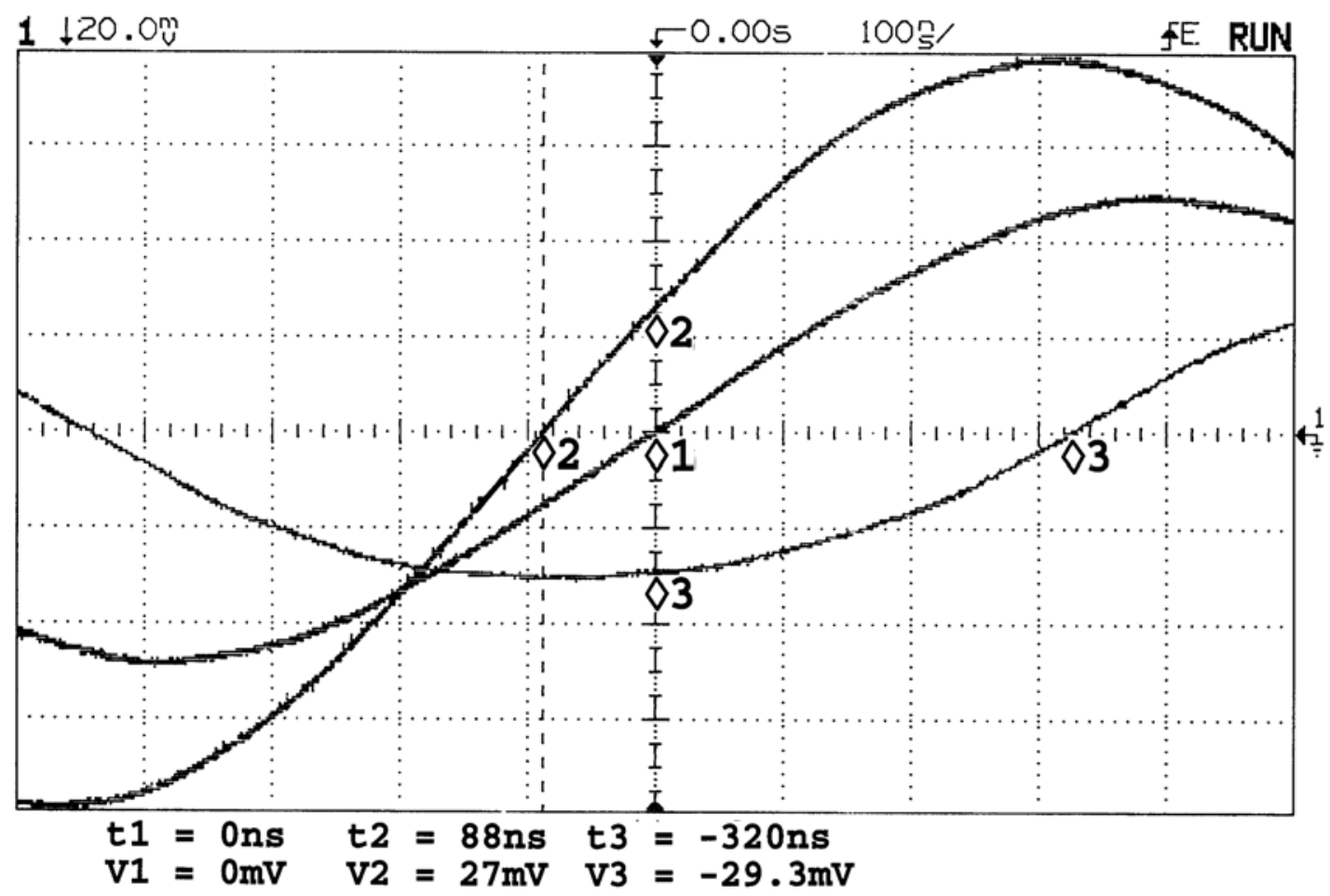

Fig. 6: Phase measurements relative to the phase of the generator: the trigger TTL signal has been adjusted to give a null voltage value, at the center of the screen, when the generator is in open circuit. The line is terminated with a matched load (plot 1), a short circuit (plot 2), and an open circuit (plot 3).

Amplitude measurements do not require time synchronization. However the measurements in Fig. 6 have been performed using the triggering functions of the oscilloscope in order to allow phase measurements relative to the phase of $\mathrm{V}_{\mathrm{g}}(\mathrm{t})$. The TTL trigger signal has been adjusted to give a null voltage at the center of the screen when the generator is in open circuit (zero phase of $\mathrm{V}_{\mathrm{g}}(\mathrm{t})$ ). The phase $<\varphi_{\mathrm{m}}$ is calculated from the time lag $\Delta t$ in the plots of Fig. 6 as

$$
\not z_{m}=\Delta t \frac{2 \pi}{T} \text { radians }=360 f \Delta \text { degrees }
$$

and the following table can be completed, where all the phases are measured taking the $\mathrm{V}_{\mathrm{g}}$ signal as reference:

\begin{tabular}{|c|c|c|c|}
\hline load & theoretic $\varphi_{\mathrm{m}}$ & measured $\Delta t$ & $\operatorname{measured} \varphi_{\mathrm{m}}$ \\
\hline $\operatorname{plot} 1 Z_{L}=Z_{0}$ & $\underline{0^{\circ}}$ & $\mathbf{t}_{1}=\underline{\mathbf{0 n s}}$ & $\underline{\boldsymbol{0}^{\circ}}$ \\
\hline plot $2 Z_{L}=0$ & $22.5^{\circ}$ & $t_{2}=\underline{88 \mathrm{~ns}}$ & $\underline{20.4^{\circ}}$ \\
\hline $\operatorname{plot} 3 Z_{L}=\infty$ & $-67.5^{\circ}$ & $t_{3}=\underline{-320 \mathrm{nS}}$ & $-74.4^{\circ}$ \\
\hline
\end{tabular}


Finally, the instantaneous voltage corresponding to the instant when the generator gives $\mathrm{V}_{\mathrm{g}}=0$ can also be determined out of Fig. 6 . Its predicted value can be calculated taking into account that:

$$
\begin{gathered}
V_{m}(t)=\left|V_{m}\right|\left\{\sin (\omega t) \cos \varphi_{m}+\cos (\omega t) \sin \varphi_{m}\right\} \\
2, \ldots
\end{gathered}=\left|V_{m}\right| \sin \varphi_{m}, \omega t=2 k \pi, \text { where } k=0,1,
$$

Now, the following table can be completed using the measured values of $\left|V_{m}\right|$ and $\varphi_{m}$ to calculate the predicted value $\mathrm{V}_{\mathrm{m}}(0)$ :

\begin{tabular}{|c|c|c|}
\hline load & predicted $V_{m}(0)$ & measured $V_{m}(0)$ \\
\hline $\operatorname{plot} 1 Z_{L}=Z_{0}$ & $\underline{0 \mathrm{mV}}$ & $\mathrm{V}_{1}=\underline{\mathbf{0 ~ m V}}$ \\
\hline $\operatorname{plot} 2 Z_{L}=0$ & $27.8 \mathrm{mV}$ & $\mathrm{V}_{2}=\underline{27 \mathrm{mV}}$ \\
\hline $\operatorname{plot} 3 Z_{L}=\infty$ & $-30 \mathrm{mV}$ & $V_{3}=\underline{-29.3 \mathrm{mV}}$ \\
\hline
\end{tabular}

$$
\mathrm{V}_{\mathrm{g}}=\underline{93 \mathrm{mV}}
$$

\section{Transmission line attenuation}

Line losses can be measured with the following method. After placing a short-circuit at the end of the line, the frequency is increased until a minimum of voltage at the input is found. At this frequency, the electrical length of the line is $\lambda / 2$. The measured voltage is null for a lossless line. However for a low loss transmission line with $\lambda / 2$ length the impedance is real and the losses can be calculated by measuring the input voltage.

$$
V_{g}=\underline{152 \mathrm{mV}} V_{\min }=\underline{16.5 \mathrm{mV}} \quad R_{e}=R_{\mathrm{g}} \frac{V_{\min }}{V_{\mathrm{g}}-V_{\min }}=\underline{6.1 \Omega}
$$

where $R_{e}$ and $R_{g}$ are the input and generator resistances respectively. Hence, the reflection coefficient $\rho_{\mathrm{e}}$ at the line input is given by:

$$
\Omega_{e}=\frac{R_{e}-Z_{0}}{R_{e}+Z_{0}}=\underline{-0.78}
$$

When $l=\lambda / 2$, the line attenuation $\alpha$ can be calculated by using the following equation, derived in the exercises previous to the laboratory work:

$$
a=\frac{-l}{2 \ell} \operatorname{Ln}\left(-, a_{e}\right)=\underline{0.0022 \mathrm{Nep} / \mathrm{m}}=\underline{1.94 \mathrm{~dB} / 100 \mathrm{~m}}
$$

This value compares very well with the attenuation plot (Fig. 3) at $1.74 \mathrm{MHz}$. 


\section{Conclusions}

This work has presented a transmission line demonstrator for intermediate level communication engineering students, which allows low cost multiplication of working benches by making use of standard instrumentation, usually available in a basic electronics laboratory (Fig. 7). The set-up allows both time-domain and sinusoidal steady state measurements. However, the paper is mainly addressed to this last which is scarce in the literature and devoted exclusively to the slotted transmission line.

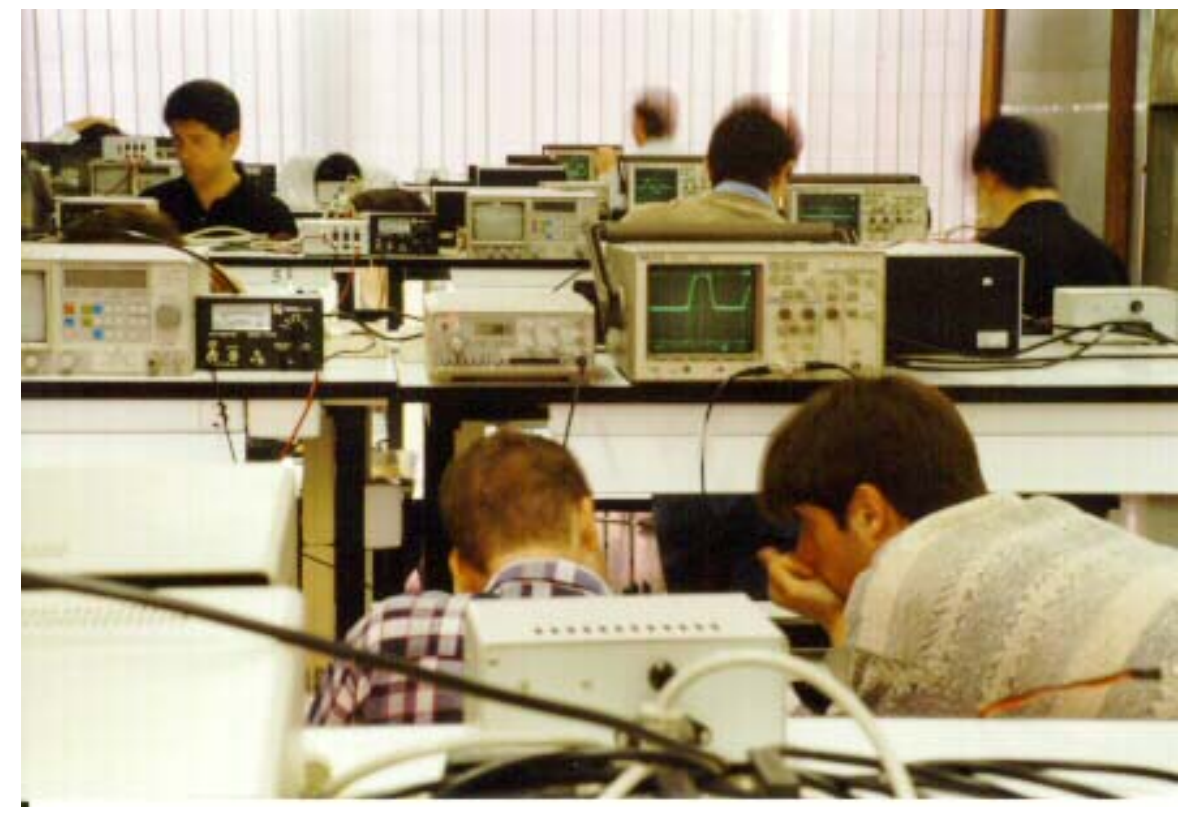

Fig. 7: General view of the laboratory

These laboratory sessions have been used to illustrate a new educational experience carried out at the Telecommunication Engineering School from the Universitat Politècnica de Catalunya (Spain). The authors have clearly distinguished between the objectives pursuit in an introductory laboratory devoted to intermediate level students and those objectives aimed at higher level students. These differences have led to a novel educational laboratory conception, and a special arrangement of the tasks to be performed by those intermediate level students.

\section{Author Contact Information}

Francesc Torres

Dept of Signal Theory and Communications

Universitat Politècnica de Catalunya

Campus Nord UPC. Edifici D3.

c/ Jordi Girona 1,3.

08034 Barcelona. Spain

Phone: 34-3-4017218 
Fax: 34-3-4017232

E-mail: torres@voltor.upc.es

Ignasi Corbella

Dept of Signal Theory and Communications

Universitat Politècnica de Catalunya

Campus Nord UPC. Edifici D3.

c/ Jordi Girona 1,3.

08034 Barcelona. Spain

Phone: 34-3-4017228

Fax: 34-3-4017232

E-mail: corbella@voltor.upc.es

Angel Cardama

Dept of Signal Theory and Communications

Universitat Politècnica de Catalunya

Campus Nord UPC. Edifici D3.

c/ Jordi Girona 1,3.

08034 Barcelona. Spain

Phone: 34-3-4016797

Fax: 34-3-4017232

E-mail: cardama@voltor.upc.es

Jordi Romeu

Dept of Signal Theory and Communications

Universitat Politècnica de Catalunya

Campus Nord UPC. Edifici D3.

c/ Jordi Girona 1,3.

08034 Barcelona. Spain

Phone: 34-3-4016826

Fax: 34-3-4017232

E-mail: romeu@voltor.upc.es

Oscar Galíndez

Dept of Signal Theory and Communications

Universitat Politècnica de Catalunya

Campus Nord UPC. Edifici D3.

c/ Jordi Girona 1,3.

08034 Barcelona. Spain

\section{Author Biographies}

Francesc Torres was born in Ibiza, Spain, in 1962. He received his Ingeniero and Doctor Ingeniero degree in Telecommunication Engineering, both from the Polytechnic University of Catalonia (UPC), Barcelona, Spain, in 1988 and 1992, respectively. In 1988-89 he was Research Assistant in the RF System Division at the European Space Agency, Holland, devoted to microwave device testing and characterization. In 1989 he joined the Antenna-Microwave-Radar group of the UPC, where he currently holds a 
post as Professor. His main research interests are focused to the design and test of microwave systems and subsystems. He is currently engaged in research on interferometric radiometers devoted to Earth observation.

Ignasi Corbella was born in Barcelona, Spain, in 1955. He received the Ingeniero and Doctor Ingeniero degrees in Telecommunication Engineering, both from the Polytechnic University of Catalonia (UPC), Barcelona, Spain in 1977 and 1983 respectively. In 1976 he joined the School of Telecommunication Engineering in Barcelona as a Research Assistant in the Microwave Laboratory, were he worked on passive microwave integrated circuit design and characterization. He became assistant professor in 1982, Associate Professor in 1986 and Professor in 1993. He is currently teaching a microwave course and is engaged in research in microwave interferometer radiometry and microwave system design in the Department of Signal Theory and Communications of UPC.

Angel Cardama, was born in Santiago, Spain, on May 13, 1944. He received the Ingeniero de Telecomunicación degree from the Polytechnic University of Madrid, Madrid, Spain, in 1968, and the Sc.M. and Ph.D. degrees in electrical engineering from Brown University, Providence, RI, in 1970 and 1973 respectively. In 1972 he joined the faculty of the E.T.S.I. de telecomunicación at the Polytechnic University of Catalonia, Barcelona, Spain, where he holds the position of Professor. His research interests range from propagation in optical fibers, high frequency aperture and array antennas, and near-field antenna scanning systems to the design of microwave imaging systems and radar antennas.

Jordi Romeu was born in Barcelona, Spain, in 1962. He received the Ingeniero and Doctor Ingeniero degrees in Telecommunication Engineering, both from the Polytechnic University of Catalonia (UPC), Barcelona, Spain in 1986 and 1991, respectively. In 1985 he joined the Electromagnetic and Photonic Engineering Group of the Signal Theory and Communications Department there. Currently he is Associate Professor at UPC, where he is engaged in research in antenna near field measurements, antenna diagnostics and antenna design.

Oscar Galíndez was born in Barcelona on March 1973. He is presently working in his last year final work to get the Communication Engineering degree at the School of Telecommunication Engineering of Barcelona, Catalonia ,Spain. 